\title{
CONVERGENCIA ENTRE EL CONOCIMIENTO, EL CONTEXTO Y LA PERSPECTIVA DE HUMANIDAD PARA LA REFORMA CURRICULAR EN LA UNIVERSIDAD LA GRAN COLOMBIA - ARMENIA: RELATO DE UNA EXPERIENCIA
}

\section{CONVERGENCE BETWEEN KNOWLEDGE, CONTEXT, AND PERSPECTIVE OF HUMANITY TOWARDS THE CURRICULUM'S REFORM AT UNIVERSIDAD LA GRAN COLOMBIA ARMENIA. AN EXPERIENCE TO SHARE}

\author{
Bibiana Vélez Medina* \\ Ximena Cifuentes Wchima** \\ Jairo Montoya López ${ }^{* \star *}$
}

Vélez M. Bibiana, Cifuentes W. Ximena, Montoya L. Jairo. Sophia N 7 - 2011. ISSN: 194-8932 Págs. 9- 19. Recepción: Junio 14 de 2011 Aceptación: Julio 30 de 2011

\section{RESUMEN}

En términos generales, se observa que los currículos en la Educación Superior del país han surgido a partir del conocimiento que determinados gremios específicos legitiman y validan como saber disciplinar necesario para la formación de los futuros profesionales. Sin embargo, la dificultad para la auto-crítica en la cual se ha sumido la Universidad moderna (Lyotard, 2000 y De Zubiría, 2007) ha conducido a que los currículos actuales sean réplicas de los problemas estructurales de organización del conocimiento mono-disciplinar y universalizante, con una consecuente fragmentación, hiperespecialización, desactualización, y poca pertinencia. Teniendo en cuenta lo anterior, la Universidad La Gran Colombia - Armenia ha adelantado un proceso de re-organización curricular soportada desde tres aspectos que dan un sustento sistémico, si se puede afirmar post-metafísico, a la estructuración académica; ellos son: el conocimiento y su episteme, el contexto en el tiempo presente y la necesidad de trazar una nueva perspectiva de humanidad. A partir de este ejercicio desarrollado a través de Comunidades Académicas para lograr una educación pertinente en el tiempo contemporáneo, se redefine el escenario de formación para nuestros estudiantes.

Como resultado de la articulación de esta triada y en analogía con las "cuencas del conocimiento" en las cuales converge cada uno de los ejes planteados, surgen nuevas condiciones de posibilidad para que la Universidad defina su rediseño curricular, y con con base en este la estructura académica, los perfiles de estudiantes y egresados, las políticas de

* Vicerrectora Académica Universidad La Gran Colombia - Armenia. Líder Grupo de Investigación PAIDEIA (Categoría A). viceacad@ugca.edu.co - Colombia.

**Decana Facultad de Ingeniería. Universidad La Gran Colombia - Armenia. defingenieria@ugca.edu.co - Colombia.

*** Secretario Académico Facultad de Ingeniería. Universidad La Gran Colombia - Armenia. secreaingenierias@ugca.edu.co Avenida Bolívar No. 7-46 Armenia, Quindío. Colombia. 
Proyección Social, Investigación y Formación, en las cuales se evidencia una postura integral que supera la mirada centrada en lo disciplinar para abordar con mayor pertinencia la responsabilidad Universitaria frente a la formación de profesionales. Este ejercicio además, nos ha permitido avanzar en la cualificación del aula como escenario primordial en el cual confluye la triada planteada, y se articula todo el proceso educativo.

\title{
PALABRAS CLAVE
}

Currículo, conocimiento, contexto, perspectiva de humanidad, cuencas del conocimiento, comunidades académicas.

\section{ABSTRACT}

\begin{abstract}
In general terms, it's been observed that curricula at universities have emerged departing from the knowledge legitimated and validated by certain groups as the disciplinary knowledge most required for professionalizing purposes. However, self-criticism crisis, in which Modern University has fallen into, (Lyotard, Zubiria) is turning current curriculums into a replica of the organization of knowledge structural problems, with the consequent fragmentation, hyper specialization, downgrading, and lack of pertinence. Taking these premises into account, Universidad La Gran Colombia Armenia, has forwarded a process towards a re organization of the curricula, to support the academic structure from a systemic and if it can be said post metaphysical approach as follows: Knowledge and its Episteme, context in current times, and the urge on tracing a new perspective of humanity. Departing from this exercise developed within the academic communities towards a pertinent education for current times, the education scenario for our students is redefined.

As the result of the articulation of this triad, and in analogy with the so called "knowledge Basins" to converge into each of the stated axes; new possible conditions come up for La Gran Colombia University to redefine the structure of curricula and based on this, the curriculum guidelines, the students and graduated profiles, the social work, research and formation policy, evidencing an integral formation surpassing the one centered on the disciplinary approach. This exercise has helped to qualify methodologies in the classroom which is the primary scenario to convey the stated triad and articulating the whole educational process.
\end{abstract}

KEY WORDS

Curricula, Context, Knowledge, perspective of humanity, knowledge basins, Academic Communities.

\section{INTRODUCCIÓN}

Como punto de partida es preciso reconocer que la característica fundamental de los currículos clásicos ha sido la hiper-especialización del conocimiento, con la consecuente fragmentación del saber en micro-objetos de estudio, que el currículo traduce en asignaturas. Con ello, la visión global y transdisciplinaria de las ciencias se reduce a un monólogo epistémico y condiciona las posibilidades de humanización que el mundo de hoy enfrenta en la formación de profesionales. De esta manera, los saberes producidos se condensan en istmos aislados, y la interrelación mínima necesaria para propiciar la interdisciplinariedad, queda supeditada a encuentros eventuales, en los cuales se defiende con mayor arraigo la postura del saber dominante, generando segregación, delimitación, y comprensión parcial por la especificidad.

Esta mirada del conocimiento ha permeado la construcción curricular en las Universidades del País, por tanto, la validación de los procesos formativos ha sido el resultado de la mirada casi exclusiva, frente a la disciplina, con lo cual el currículo no solo ha perdido su carácter integrador y sistémico, sino que además ha ido perdiendo pertinencia e impacto para la transformación de la humanidad.

En este contexto, no es extraño encontrar expresiones de reclamo cuando la sociedad se pronuncia frente a la academia, juzgando sus procesos como asilados de la realidad e ignorantes de las expectativas y las necesidades del país. Pero el asunto supera a los gremios disciplinares, ya que debe ubicarnos frente al análisis de nuestra actuación, frente a la responsabilidad que tenemos en el momento histórico-crítico, por el cual atraviesa la humanidad.

Teniendo en cuenta lo anterior, la Universidad La Gran Colombia - Armenia (UGCA) ha venido construyendo un ejercicio de re-diseño curricular soportado no solo desde la mirada de las diferentes disciplinas, sino a partir de lo que hemos denominado Cuencas del Conocimiento, como analogía de la cuenca hidrográfica, ellas son entendidas como un lugar de confluencia de tres caudales: el conocimiento y su episteme, el contexto en el tiempo presente y la necesidad de trazar una 
nueva perspectiva de humanidad; pero a su vez, la cuenca implica un lugar de nacimiento de algo creado a partir de la convergencia:

Figura 1. Articulación Cuencas del Conocimiento

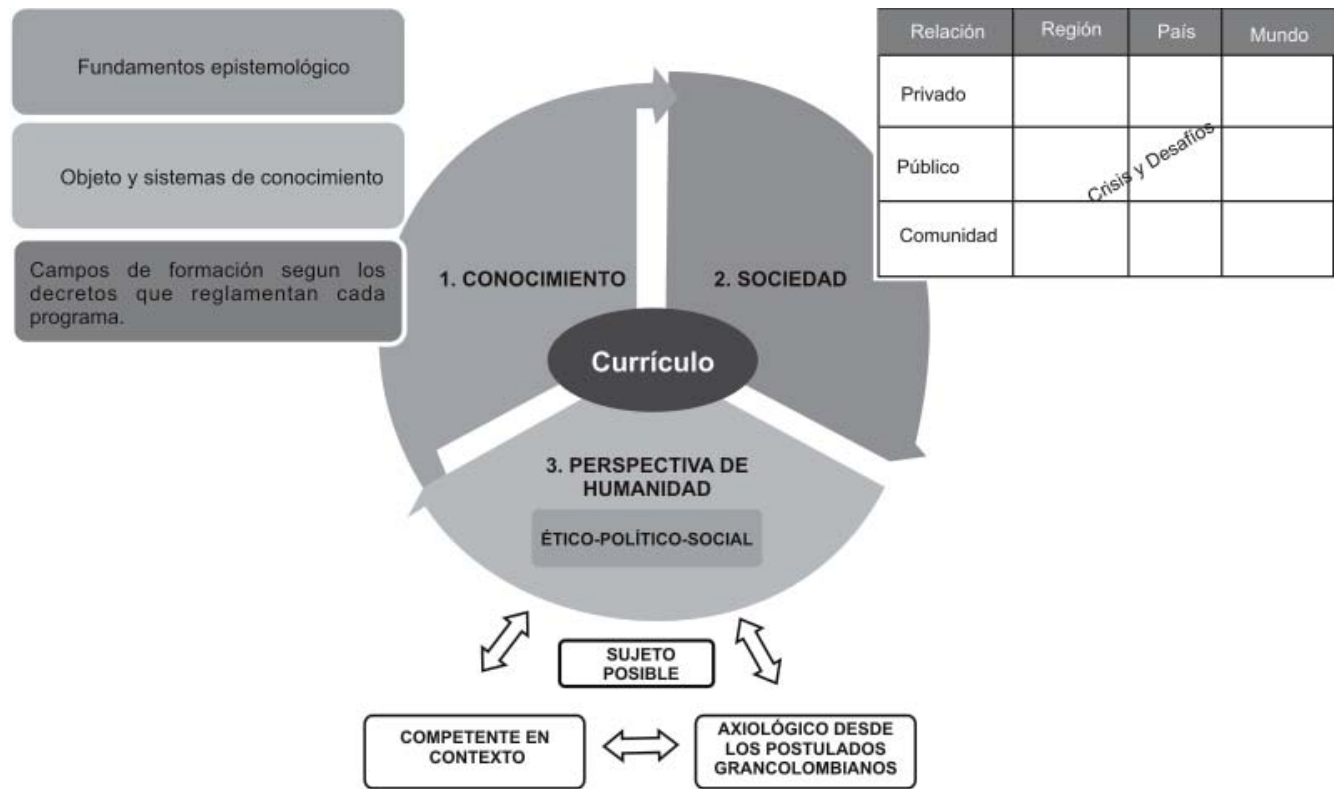

Fuente. Los Autores, 2010

\section{RUTA TRAZADA PARA CONFIGURAR LAS CUENCAS DEL CONOCIMIENTO}

Sin el afán por intentar definir asignaturas, intensidades horarias o lugar dentro de la estructura de formación, el proceso inicial se centró en la configuración del lugar de sentido compartido por la comunidad académica. La ruta que a continuación se presenta fue trabajada por los diferentes programas académicos de la Universidad: Arquitectura, Derecho, Ingeniería Agroindustrial, Economía, Contaduría y Administración de empresas. Sin embargo, para ejemplificar el presente artículo se tomarán apartes de los resultados del programa de Ingeniería. Como un ir y venir, se tejieron conversaciones iniciales en torno a:

\subsection{Primer eje o caudal - el conocimiento-}

En este eje se retorna a la pregunta fundamental por el sentido de la disciplina: ¿qué significa ser ingeniero, abogado o arquitecto? ¿Para qué formar en la economía, la administración o la contaduría? ¿Qué sentido tiene la disciplina en el mundo de hoy?, con ello se construye una nueva episteme, o más bien, un nuevo lenguaje e interpretación acerca del conocimiento disciplinar, sin desconocer aquellas bases fundantes que ya han sido convalidadas y legitimadas por las diversas comunidades académicas. En este momento de la cuestión, no basta con la pregunta uni-disciplinar que orienta la defininición del objeto del conocimiento, sino que se debe pensar la disciplina desde su complejidad, lo que implica superar el cuestionamiento por el "objeto de estudio" y pensar los "sistemas" que lo comprenden (Luhmann), con ello tendríamos una mirada complementaria de los retos interdisciplinares y transdisciplinares, que para la ingeniería, el derecho, la arquitectura, la administración y los demás programas, suscitaría interrogantes de problematización como: ¿Con qué otros sistemas de conocimiento debe interactuar el economista o el abogado Gran Colombiano? y ¿Cuáles son los nuevos objetos de conocimiento que surgen al interactuar con otras disciplinas? 
Finalmente, en este eje o caudal también se incluye la pregunta por los campos de formación reglamentados a partir del objeto y los sistemas, requeridos en la formación de los profesionales.

El camino a seguir para la constitución del primer eje es el siguiente:

a) Retornar al fundamento epistémico de la disciplina, con especial atención hacia las tendencias contemporáneas que marcan los nuevos paradigmas.

b) Definir brevemente el objeto de conocimiento que dio origen a la disciplina.

c) Definir los sistemas de conocimiento interdisciplinares con los cuales interactuará el objeto de conocimiento.

d) Identificar los campos de formación definidos en los decretos que reglamentan cada disciplina.

e) Identificar los campos de formación definidos por las asociaciones disciplinares, en la actualidad.

f) Identificar los campos de formación definidos Internacionalmente, que se implementan en Universidades de reconocida trayectoria en el Mundo.

\subsubsection{Objeto de conocimiento de la disciplina} Uno de los aspectos más olvidados en Colombia y en América Latina tiene que ver con el objeto de conocimiento de las diversas disciplinas, preguntas como: ¿para qué estudiar determinada disciplina? o ¿qué estudia cierta disciplina? son preguntas que fundamentan el ejercicio de la profesión, pero han quedado rezagadas por el tecnicismo, la burocratización del saber y la hiper-especialización. La sociedad del conocimiento ha pretendido ser tan práctica, que ha olvidado los sentidos y significados que fundamentan el que hacer profesional, como si el ser y el conocer de lo disciplinar no surgiera de un verdadero amor por el conocimiento, o dicho en términos de Nietzsche, como si: "Ios prácticos prosaicos carecieran de ideas precisamente, $y$, por eso, carecieran también de una praxis auténtica" (Nietzsche, 2000). Con la pregunta por el objeto no se pretende una mirada cartesiana, por el contrario, se busca un retorno místico a los lenguajes, experiencias y sentidos teleológicos que originaron cierta actividad humana, para intentar recuperar el sentido perdido en estos tiempos de modernidad líquida (Bauman, 2005) en que se carece de todo fundamento.

Las preguntas básicas han quedado olvidadas en los currículos de las universidades, pues la construcción curricular ha pasado a ser una administración de asignaturas, que muchas veces carece de significado. ¿Qué pasa entonces con este tipo de currículos? la poca reflexión curricular desde la complejidad sistémica, conlleva a que el estudiante curse una cantidad de asignaturas de manera fragmentada y sin un sentido integrador, ni comprensivo de la disciplina. Para superar la partición del objeto de estudio y su hiperespecialización se debe retomar la pregunta por el sentido del conocimiento que se interrelaciona en cada una de las disciplinas, y acercarnos al cuestionamiento que fundamenta la episteme contemporánea, a saber: ¿qué sentido tiene conocer?

En esta dirección, los encuentros realizados por la comunidad académica de los diferentes programas de la UGCA configuraron sus objetos de estudio, desde el acuerdo de mínimos compartidos. Para el caso de Ingeniería quedó expresado de la siguiente manera:

"La facultad reconoce al ingeniero como un profesional cuyo escenario de trabajo es el contexto disciplinar y que fundamentado en la investigación, la innovación y el análisis; diseña, procesa e implementa soluciones de tal manera que sean herramientas que den respuesta a problemas de diferentes ámbitos, orientando su trabajo hacia los procesos de desarrollo, bajo los criterios de competitividad y responsabilidad social" 1

\subsubsection{Sistema de conocimiento}

En la contemporaneidad no es posible referenciar única y exclusivamente el objeto de conocimiento de una disciplina; de ahí que el mayor desafío de la innovación curricular radique en la necesidad de superar la indagación del Objeto por la del Sistema de Conocimiento (Morin, 1997). En la transposición señalada juega un papel importante el diálogo entre diferentes saberes, pues sólo desde la interacción de diversas

1 Facultad de Ingeniería. Universidad La Gran Colombia Armenia. Talleres para la construcción curricular. Armenia, 2010. 
disciplinas es posible re-crear y de-construir el saber contemporáneo. Ahora bien, para pensar el sistema de conocimiento de las disciplinas tendríamos que preguntarnos: ¿Qué se requiere interdisciplinariamente para ser ingeniero o contador o administrador en el mundo contemporáneo? ¿Con cuáles disciplinas debe conversar la ingeniería para resolver los problemas más críticos del presente? Estos interrogantes ponen de manifiesto, que a pesar de los avances de la ciencia en la modernidad, el mundo contemporáneo no es un mundo fragmentado, ya que en la actualidad nos enfrentamos a la solución de problemas que no tienen respuesta exclusiva en la disciplina, problemas como el agua, la pobreza, las migraciones forzosas, la destrucción de ecosistemas o la violencia, necesitan una mirada interdisciplinaria que posibilite la solución en consenso desde varios saberes, para ello la pregunta que orienta la fundamentación curricular debe ser: ¿Con cuáles sistemas de conocimiento interactuará el objeto de conocimiento en cuestión para resolver los grandes conflictos del mundo de la vida?

\subsubsection{Campos de formación}

Los campos de formación hacen referencia a aquellos que han sido legitimados por los gremios o asociaciones de las profesiones. En Colombia han sido regulados y estas regulaciones obedecen a acuerdos definidos por las comunidades disciplinares quienes establecen las áreas de formación mínimas que deben incorporar los planes de estudio de cada programa académico. Dado que es un componente relacionado con la legalidad para el funcionamiento, debe ser incluido en el análisis del primer eje de la triada.

\subsection{Segundo eje o caudal - el contexto -}

¿Qué significa pensar el contexto hoy? Significa ante todo volver la mirada sobre los grandes y complejos problemas que existen en la humanidad. Para ello es necesaria la pregunta: ¿cuáles son los problemas que a pesar de los avances científicos, tecnológicos y del conocimiento en general, no han sido solucionados todavía por el hombre? ¿Qué ocurre y transcurre en el mundo actual? De ahí que la pregunta por el contexto deba ser abordada con una mirada crítica sobre el tiempo presente sin ignorar, o pretender ocultar, las verdaderas crisis aún no resueltas en el siglo XXI.
Para nuetro ejemplo no solo se debe preguntar: ¿Cuáles son los problemas de la Ingeniería hoy?, sino dar una mirada global del mundo, el país y la región, para comprender cómo la ingeniería ha sido ignorante frente a la crisis en algunos contextos, mientras que en otros ha posibilitado salidas y nuevos cauces. Ante todo tendríamos que preguntarnos como sujetos de conocimiento: ¿Cuáles son los retos de la ingeniería en el contexto y en el tiempo presente?

La perspectiva propuesta, más que ser una mirada innovadora es una mirada de responsabilidad social contemporánea, pues las universidades no pueden seguir pensando que son centros de especialización, sino que deben presentar visiones más amplias y cercanas al real mundo de la vida para tratar de integrar los asuntos cotidianos, los problemas del contexto, y los problemas del conocimiento.

En tal sentido, al menos tres aspectos específicos del contexto deben analizarse: lo público, lo privado y la comunidad. El primero de ellos se aborda con preguntas como ¿qué está pasando en lo público? ¿qué está pasando a nivel del estado? ¿qué está pasando a nivel de las regulaciones constitucionales? y ¿qué esta pasando entre la relación estado-gobierno?; al segundo aspecto le compete preguntar: ¿qué está pasando en lo privado? ¿qué ocurre con la empresa hoy? ¿qué ocurre con los monopolios hoy? ¿porqué el estado perdió el carácter legitimador del conocimiento? y sobre todo ¿quién legitima el conocimiento actualmente? ¿Por qué el mundo se volcó hacia las grandes multinacionales como el gran movimiento que regula lo estatal, lo político, y las tendencias en el mundo? (Max Neef, 2006), en relación con lo privado y los sectores de producción deberíamos preguntar además: ¿qué está pasando con los medios de comunicación? ¿hacia donde nos lleva la mirada de los medios de comunicación? y ¿qué paradigma está trazando la sociedad contemporánea a partir de la información que nos llega desde la mirada de los medios de comunicación?; por último, en un tercer nivel la pregunta es: ¿que está pasando con la comunidad? ¿cuáles son las crisis del ciudadano común?, ¿cuáles son las problemáticas de los grupos vulnerables y excluidos?, pues los factores de lo público y lo privado no dan cuenta absoluta de lo que ocurre en la comunidad, ya que en la comunidad se 
observan los problemas reales del contexto, y se enfatiza el corto impacto de las universidades en la transformación social.

El siguiente cuadro explicita y problematiza este eje de la cuenca:

\begin{tabular}{|c|c|c|c|}
\hline Relación & Región & País & Mundo \\
\hline Privado & & & \\
\hline Público & crisi & & \\
\hline Comunidad & & & \\
\hline
\end{tabular}

Fuente: Vélez, 2010

Lo que se propone en la UGCA es que la mirada de la comunidad, es decir la mirada del contexto cercano, de la lógica de la vida cotidiana y común, tiene que permear directamente el currículo y la formación de los estudiantes, pues allí es donde está la profunda responsabilidad social que tienen las universidades de la región y del país. Cuando el egresado interactúa con el medio, cuando se enfrenta al contexto del mundo de la vida no solo lo hace a partir del conocimiento, sino ante todo desde la responsabilidad civil y ciudadana que le fomentó la universidad y desde la mirada crítico social que alcanza del contexto. Valdría la pena recordar que "los hombres son hombres, antes que médicos, ingenieros o arquitectos. (Por tanto,) la educación deberá hacer de ellos hombres honestos, sensatos y capaces, y ellos con el tiempo se transformaran en médicos, ingenieros y arquitectos, honestos, sensatos y capaces" (Jhon Stewart Miller, 2003).

Pero, desde el contexto no solamente tiene que existir una mirada crítica sino una mirada de esperanza en la cual se trace el norte hacia dónde se debe movilizar la sociedad. La universidad en sus orígenes era quien movilizaba a la sociedad desde sus postulados de desarrollo y progreso, sin embargo hoy en día la universidad ha perdido la batuta y va tras lo que socialmente acontece. En este sentido, debemos recuperar la voz que anuncia y propicia la verdadera transformación social, luego, la UGCA en sus currículos está llamada a recuperar la esperanza de un futuro posible para todos, una sociedad de realizaciones humanas en la cual se evite la exclusión, la violencia y la desolación actual.

\subsection{Tercer eje o caudal - Perspectiva de Humanidad -}

La perspectiva de humanidad en la UGCA intenta trazar un desafío sobre la consciencia ética ciudadana y planetaria que deben tener nuestros estudiantes y egresados, ¿Qué significa ser ciudadano del planeta? ¿Qué significa ser ciudadano para la construcción de una nueva humanidad? y en el caso particular de la ingenieria ¿Será posible formar una nueva humanidad con apoyo de la Ingeniería? ¿Cuál es el sentido ético, social, político e histórico del Ingeniero? estas preguntas orientan la perspectiva de humanidad en el diseño curricular, pues la universidad puede estar bien fundamentada en lo epistémico, y bien contextualizada en lo social, pero si no reflexiona por el ser humano que quiere formar, se puede cometer el error de servir a quienes piensan a los individuos como medios y no como fines propios.

La pregunta por el tercer eje es una pregunta por la conciencia; el primer eje de la triada se pregunta por el conocimiento, el segundo por la responsabilidad social en contexto y el tercero por la conciencia ética, ciudadana y planetaria que deben tener nuestros estudiantes, para el despertar de una nueva moral acorde con el mundo contemporáneo.

Sin embargo, formar una conciencia planetaria, una conciencia ciudadana autónoma, debe ser tenido en consideración cuando se apuesta por un currículo diferente, puesto que la formación en perspectiva de humanidad, no es solamente una perspectiva ética, sino que además es una perspectiva multicompetente, lo cual implica saber hacer con el sistema de conocimiento, con el objeto de conocimiento, con el campo de formación, pero sobre todo: saber quién soy en el mundo, en la nación, en la región y como actuó frente a lo público, lo privado, y la comunidad. 


\section{RUTA PARA EL RE-DISEÑO CURRICU- LAR: CONVERGENCIA Y DESPLIEGUE DE LAS CUENCAS DEL CONOCIMIENTO}

La articulación de la triada fundamenta los principios básicos para el re-diseño curricular a partir de los cuales se abordan los siguientes niveles de desarrollo, es decir, el plan de estudios y el micro-currículo.

Para el Diseño del Plan de Estudios, es necesario en primer lugar definir a partir de las cuencas el perfil del programa y el perfil del egresado expresado en competencias. Es decir, cuál es el perfil del programa y del egresado desde el eje del conocimiento, el contexto y la perspectiva de humanidad.

\subsection{La deconstrucción del perfil profesional: punto de partida para la articulación en- tre currículo, investigación y proyección Social}

Teniendo en cuenta las crisis, desafíos y responsabilidades que el mundo del tiempo presente traza a la educación superior, relacionadas con la formación de sujetos capaces de transformar las realidades humanas marcadas por la desigualdad, exclusión y marginalidad; pero a la vez, sujetos virtuosos para la creación, innovación y contribución al desarrollo social, el debate actual para la formación de profesionales y la planeación curricular debe girar en torno a la pregunta por el perfil de los egresados que se enfrentan a la complejidad de un mundo en cual tienen el reto de trascender.

En tal sentido, el punto de partida para abordar la reforma curricular, investigativa y social de los diferentes programas de la UGCA ha sido el planteamiento inicial sobre el alcance que se pretende dar a los desempeños del egresado, de tal manera que las discusiones se concentraron, a partir de las cuencas del conocimiento, en establecer un perfil como umbral de posibilidad, rasgo distintivo y cualidad innovadora de la formación profesional.

No es posible concebir este ejercicio sin la anuencia del equipo de docentes, estudiantes y egresados del programa, quienes se encuentran en la discusión colegiada a través de la configuración de Comunidades Académicas como escenario para dar sentido compartido, protocolización de acuer- dos y definición de fundamentos, que articulan sin unificar el pensamiento, pero que en definitiva apalancan la construcción de los programas académicos ideados. A manera de movimientos no instruccionales, sino orientadores, se presenta a continuación la ruta seguida para la concepción del perfil profesional de los programas, ejemplificando en algunos casos a partir del proceso de Ingeniería Agroindustrial.

\subsubsection{Movimiento uno: definición de Fundamentos del Perfil}

Los ejes en torno a los cuales giró este primer movimiento fueron las tres grandes categorías de problematización expuestas anteriormente: Conocimiento - Contexto - Perspectiva de Humanidad, desde los cuales se logró focalizar un perfil de programa que involucrara articuladamente los sentidos de formación del abogado, el arquitecto o el ingeniero, los contextos (reales e ideales) en que se desenvuelve, reconociendo los desafíos y las características humanas, éticas, ciudadanas y morales desde las cuales se ejerce el quehacer profesional de la ingenieria.

\subsubsection{Movimiento dos: \\ definición del perfil de programa}

El análisis categorial de los tres insumos anteriores (conocimiento, contexto y perspectiva de humanidad) permite la definición del Perfil del Programa entendido como la identidad estratégica, misional y prospectiva que caracteriza y diferencia al programa académico, del resto de programas del país. El perfil profesional debe incluir su postura frente al manejo, conservación y renovación del conocimiento propio de la disciplina y la relación con la interdisciplina; así mismo, en él se plantean los desafíos de actuación pertinente y la prospectiva en el contexto, que fue observada, analizada y discutida por toda la comunidad académica en mensión; y finalmente, la posición ética frente a la perspectiva de humanidad propia del programa.

De esta manera quedó expresado cada uno de los perfiles, como ejemplo se presenta el de Ingeniería Agroindustrial: "El programa de Ingeniería Agroindustrial de la Universidad La Gran Colombia forma ingenieros innovadores y éticos con gran capacidad de liderazgo, reflexión permanente y compromiso con el desarrollo rural y la integración agroindustrial sostenible de la región y el país, fortaleciendo y consolidando los procesos 
tecnológicos e investigativos en una dinámica permanente de construcción de conocimiento. El programa forma profesionales integrales e idóneos con un alto grado de motivación hacia el aprendizaje que, ubicados desde la posproducción, son capaces de liderar, desarrollar y administrar procesos de innovación en el ámbito de las cadenas productivas, los cuales permiten consolidar procesos de investigación para fortalecer el tejido social y generar desarrollo sostenible en la región y el país" (PEP, 2011).

\section{Perspectiva de humanidad}

El programa de Ingeniería Agroindustrial de la Universidad La Gran Colombia forma ingenieros innovadores y éticos con gran capacidad de liderazgo, reflexión permanente y compromiso con el desarrollo rural y la integración agroindustrial sostenible de la región y el país (...)

Fuente: Facultad Ingeniería UGCA, 2010

\subsubsection{Movimiento Tres: \\ Definición del Perfil del Egresado}

A partir de la concepción del Perfil de los Programas de la Universidad, las distintas Comunidades Académicas emprendieron la construcción del Perfil Del Egresado. El rasgo diferenciador entre un perfil y otro, se fundamenta en que el segundo (perfil egresado), se expresa en términos de competencias, habilidades o desempeños específicos que permiten al profesional ejercer el despliegue de sus conocimientos en un contexto de problemas y oportunidades en el cual se expande su potencialidad. Teniendo presente que una competencia es la capacidad de hacer uso inteligente y apropiado de un Conocimiento en un Contexto determinado y bajo unos principios Éticos, la relación entre ambos perfiles podría expresarse de la siguiente manera:

Así, el modelo y la fundamentación para la formulación del Perfil del Egresado tuvo como soporte la definición de aquellas competencias que la comunidad académica consideró como el máximo ideal para la formación, las cuales están relacionadas

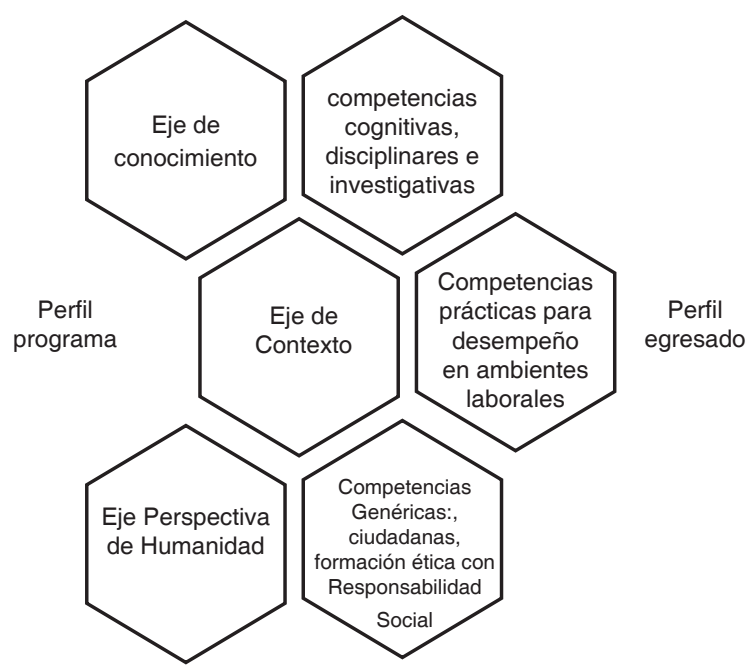

Fuente: Vélez, 2010 
con el análisis de conocimiento requerido en la disciplina e interdisciplina, el contexto, así como la perspectiva humana.

\subsubsection{Movimiento cuatro: estructuración del currículo}

Una vez se ha definido de manera concertada el Perfil del Egresado, es posible iniciar la reforma curricular. Vale la pena anotar que en este modelo de reforma, no se partió del análisis directo y aleatorio sobre asignaturas, ni tampoco se inició el proceso desde teorías pedagógicas, sino que la renovación del currículo partió del punto de llegada del ideal de formación, el cual pasó por el sustento epistémico, contextual y humano como soportes que garantizan la pertinencia, actualidad y conexión con las realidades del mundo del tiempo presente. Entendido como sistema, podría diagramarse de la siguiente manera:

\begin{tabular}{|l|l|l|l|}
\hline \multicolumn{2}{|c|}{ Insumos } & Procesos & Productos \\
\hline FUNDAMENTOS & CONOCIMIENTOS & PLAN DE & $\begin{array}{l}\text { Perfil del } \\
\text { egresado }\end{array}$ \\
Filosóficos & & ESTUDIOS & \\
Epistemológicos & SOCIEDAD & & \\
Psicológicos & & & \\
Pedagógicos & & Perfil \\
Sociológicos & Perspectiva de & & requaciobnal \\
Polítocos & Humanidad & & \\
Ambientales & requerido \\
Admnistrativos & & & \\
Internacionales & & & \\
\hline
\end{tabular}

Fuente: Escuela de Pedagogía UGCA, 2010

Con el perfil definido se organizan entonces los campos, componentes y subcomponentes necesarios para dar cumplimiento a las competencias definidas para el profesional y luego se procede a fijar las competencias por áreas (máximo ideal de formación por área) que se articulan con el perfil del egresado, así:

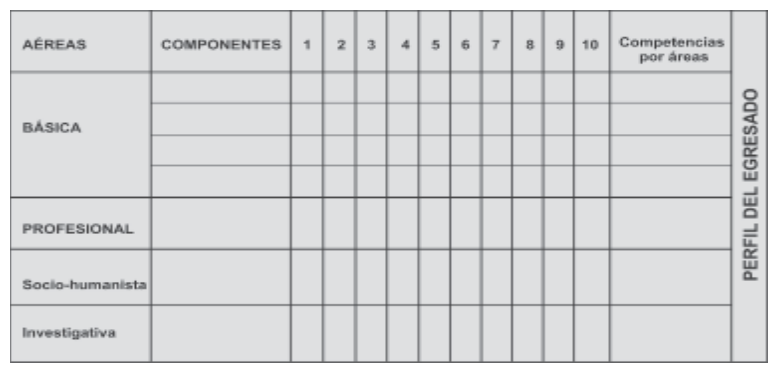

Fuente: Escuela de Pedagogía UGCA, 2010
Como se mencionó anteriormente, el proceso de rediseño curricular de los programas de la UGCA no ha sido un ejercicio aislado de ubicación aleatoria de asignaturas, sino por el contrario un desarrollo sistémico de los lineamientos trazados por la institución, para tal fin el currículo ha sido abordado desde tres parámetros de consistencia, a saber: consistencia horizontal, vertical y transversal, para su concreción en los procesos de aula.

\subsection{Pasos para construir el plan de estudios}

\subsubsection{Consistencia Horizontal}

El plan de estudios de cada programa se diseñó en primer lugar desde la lógica horizontal estructurada por áreas y componentes, a partir de las cuales se desarrollan de manera progresiva y secuencial las diferentes asignaturas que apuntan al logro de competencias articuladas al perfil del programa.

Aprender es adaptarse, y como tal, supone dos aspectos indisociables: la asimilación y la acomodación (Piaget, 1933). Es así, como se plantea que toda situación de aprendizaje implica una asimilación (permite incorporar una nueva experiencia a otra ya conocida) y una acomodación (permite transformar su modelo mental y configurar un nuevo saber). Teniendo en cuenta estos planteamientos la estructura curricular de cada programa está diseñada bajo el sistema de créditos desde una lógica de secuencialidad que privilegia el aprendizaje autónomo del estudiante a medida que avanza en el desarrollo de su programa académico, de tal manera que a través del proceso formativo se van logrando las estructuras cognitivas a partir de la adquisición gradual de competencias que apuntan todas al logro de un fin común, esto es: El Perfil del Egresado.

Teniendo en cuenta la premisa anterior, se seleccionaron las asignaturas adscritas a cada área, componente y subcomponente desde la lógica de aprendizaje gradual y secuencial que aumenta en complejidad. A cada asignatura se le asignó un número de créditos dependiendo de la necesidad de intensificar o disminuir la presencia del docente para la adquisición de competencias. La definición del número de créditos académicos para cada una de las asignaturas del plan de estudios se fundamentó desde el punto de vista de la psicología evolutiva planteada por Piaget 
(1933), la cual establece que las estructuras cognitivas para la adquisición del aprendizaje cambian en el tiempo, configurando etapas del desarrollo, donde cada etapa guarda una disposición temporal móvil, sin importar demasiado la edad en que cada una de ellas se presenta, pero con la condición de posibilidad de que se integren naturalmente en las etapas posteriores. Así mismo, se privilegió el desarrollo progresivo del aprendizaje autónomo del estudiante.

\subsubsection{Consistencia Vertical}

Una vez definidas las asignaturas requeridas en el plan de estudios, articuladas por una lógica horizontal de secuencialidad, es necesario avanzar hacia un siguiente nivel de organización, consistente en superar la idea de asignaturas aisladas en los semestres, las cuales se deben articular para dar sentido integrador al aprendizaje simultáneo de diferentes conceptos. De esta manera, el programa concibió el desarrollo de Proyectos Integradores como estrategia para cohesionar la lógica de la estructura vertical.

Los Proyectos Integradores se estructuran y articulan desde las necesidades de énfasis de formación trazados en el Perfil del Programa, en el caso de Ingeniería Agroindustrial, los proyectos se organizaron por ciclos desde tres énfasis estratégicos surgidos del Eje de Contexto, ellos son: Biotecnología, Sistemas de Gestión de Calidad y Desarrollo Rural. Para lograr una mayor consistencia vertical cada proyecto se desarrolla por ciclos que agrupan tres o cuatro semestres, y se articulan desde el área de formación investigativa que atraviesa desde el primero hasta el último semestre del plan de estudios.

\subsubsection{Consistencia Transversal}

Finalmente, se retomó el Eje de Perspectiva de Humanidad para alcanzar las competencias trazadas en el perfil Grancolombiano. De esta manera, el desarrollo de la lógica transversal, se dio a partir de procesos no expresados en asignaturas concretas, sino de manera genérica para el logro de competencias ambientales, ciudadanas, científicas, lógicas y comunicativas, en ambientes permanentes de aprendizaje, que hallan además un espacio común en el área de formación sociohumanística.

\subsection{Concreción del Plan en Micro-currículos}

El logro de las competencias y propósitos de formación de manera secuencial a través de las diferentes áreas, componentes y asignaturas, se expresó de forma particular y específica en los Micro-Currículos, los cuales garantizan que el ideal trazado se concreta en el aula, por tal razón, en ellos se evidencia el desarrollo de:

- Relación de la asignatura con el perfil del egresado

- Relación de la asignatura con las competencias del área

- Competencias que debe desarrollar la asignatura

- (competencias disciplinares y competencias genéricas)

- Propuesta de contenidos

- Indicadores de competencias (disciplinares y genéricas)

- Evaluación por competencias

- Perfil del docente requerido para el desarrollo de la asignatura

\subsection{Articulación con la Investigación y la Pro- yección Social}

La definición del perfil concentra y enfoca los esfuerzos investigativos y el desarrollo de procesos de extensión, para el logro de un fin común. Todo el proceso de consistencia curricular implica un despliegue detallado de secuencialidad, coherencia, integralidad y flexibilidad que ha sido soportado en la construcción sistémica del Proyecto Educativo de Programa (PEP) con el fin de articular no sólo el proceso curricular al perfil del egresado, sino además los avances investigativos del programa y las metas de proyección social del mismo.

Hoy damos fe que el modelo desarrollado e implementado por la UGCA en cada uno de los programas académicos ha permito alcanzar logros significativos y reconocimientos locales y nacionales en cuanto a: productos investigativos, cualificación de grupos y semilleros de investigación, posicionamiento nacional en resultados Saber - Pro, reconocimientos y vinculaciones estratégicas con la Empresa y el Estado y una actuación más significativa y pertinente con la Comunidad. 


\section{CONCLUSIONES}

Una de las primeras ideas derivadas del proceso de rediseño curricular de los programas de la UGCA afirma la complejidad del aprendizaje, ya que se hace imposible redefinirlo separado de lo académico, lo social y lo político; de allí el resultado significativo de las Cuencas del Conocimiento y su convergencia, entre el conocimiento, el contexto y la necesidad de una nueva perspectiva de humanidad.

En la indagación por la complejidad del currículo y la necesidad de innovaciones curriculares por parte de la universidad se evidencio que el currículo en la educación superior necesita de la interacción con la investigación y la proyección social y desde luego, con alianzas estratégicas, como las propuestas por los sectores productivos y el estado, para de esta manera desplegar el currículo al entorno local y global.

Es preciso anotar que como resultado de esta articulación la universidad no sólo definió una nueva estructura académica, sino que además trazó los lineamiento curriculares, la redefinición de políticas de proyección social, políticas de investigación y políticas de formación en las cuales se ve la convergencia de las cuencas del conocimiento, así mismo se logró la redefinición de los planes de estudio, la creación de la figura: docentes asesores académicos, y ante todo la configuración de una mirada diferente sobre el aula donde converge todo el proceso.

Finalmente, se debe centrar la atención en la posibilidad interdisciplinar del los nuevos currículos, es decir, se debe transitar hacia una mayor flexibilidad curricular, pues la complejidad subyacente de las cuencas del conocimiento conduce a la comprensión y valoración de lo múlti/inter/trans/disciplinar.

\section{REFERENCIAS BIBLIOGRÁFICAS}

\section{Artículos de revistas}

Berrio, A. C. and Perez, S.J. Towards a new concept on engineering education. Journal of Educational Technology, Vol. 24, No. 12 , 2002, pp. 269-286.

DE ZUBIRÍA SAMPER, Sergio. Universidad, crisis y Nación en América Latina. En: Otras Voces. Revista de Estudios Sociales no. 26, abril de 2007: Pp. 1-196. ISSN 0123-885X: Bogotá, Colombia; Pp. 148-157.
PIAGET, J. "Psychologie de l'enfant et l'enseignement de I'histoire", en Bulletin trimestriel de la Conférence Internationale pour l'enseignement de l'histoire, 2, París, 1933.

VÉLEZ, Bibiana. Problematización: Lo humano deviene en la pregunta. En: Revista de investigación SOPHIA. Universidad la Gran Colombia. Ed. No. 5. Año 2009. P. 149-160

\section{Libros}

ACOSTA, J. Ciudades del Conocimiento. Panamericana formas e Impresos, Bogotá, D.C., 2001. 116 pp.

BAUMAN, Z. (2005). Los retos de la educación en la modernidad líquida. Barcelona: Gedisa.

CASTORIADIS, Cornelius. El campo de lo social histórico. Filosofía-historia-letras. Primavera, 1986.

LYOTARD, Jean - Fran ois. La condición postmoderna. 7 ed. Madrid: Cátedra Teorema, 2000.

MORIN, Edgar. El método II: Del objeto al Sistema. Ediciones cátedra, 1997.

NIETZSCHE, F. Sobre el porvenir de nuestras instituciones educativas. Primera conferencia.

Traducción de Carlos Manzano publicada por Tusquets, Barcelona, septiembre de 2000.

\section{Memorias de congresos}

[2] Eppinger S.D. and Salminen V.K., Patterns of product development interactions. Proceedings of ICED '01, Vol. 1, Glasgow, 2001, pp.283-290.

MAX NEEF, M. Buscar la verdad en un océano de mentiras. Trascripción de la conferencia ofrecida por el autor en el acto de Apertura del VIII Simposio de Investigación de la Facultad de Salud (25 de octubre de 2006)

\section{Fuentes electrónicas}

University of Hong Kong. (1997, June). Final report: Ad Hoc Group for Learning Technologies. Consultado el 21 de mayo de 2002 en http://www.hku.hk/caut/Homepage/itt/5_ Reports/5_1AdHoc.htm 